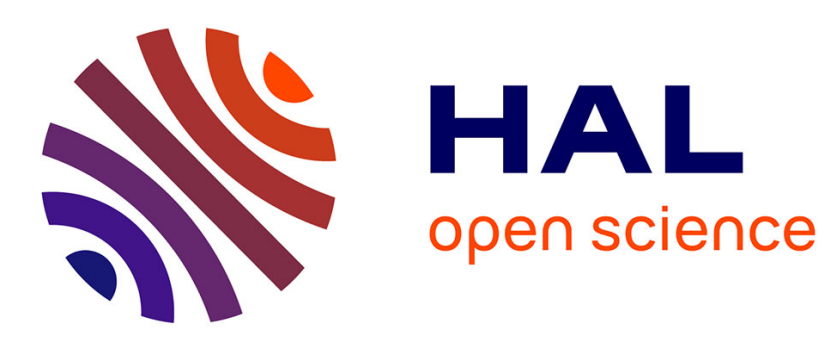

\title{
Pulsed magnetohydrodynamic blood flow in a rigid vessel under physiological pressure gradient
}

Dima Abi Abdallah, Agnès Drochon, Vincent Robin, Odette Fokapu

\section{To cite this version:}

Dima Abi Abdallah, Agnès Drochon, Vincent Robin, Odette Fokapu. Pulsed magnetohydrodynamic blood flow in a rigid vessel under physiological pressure gradient. Computer Methods in Biomechanics and Biomedical Engineering, 2009, 12 (4), pp.445-458. hal-01083975

\section{HAL Id: hal-01083975 \\ https://hal.science/hal-01083975}

Submitted on 18 Nov 2014

HAL is a multi-disciplinary open access archive for the deposit and dissemination of scientific research documents, whether they are published or not. The documents may come from teaching and research institutions in France or abroad, or from public or private research centers.
L'archive ouverte pluridisciplinaire HAL, est destinée au dépôt et à la diffusion de documents scientifiques de niveau recherche, publiés ou non, émanant des établissements d'enseignement et de recherche français ou étrangers, des laboratoires publics ou privés. 


\title{
Pulsed Magnetohydrodynamic blood flow in a rigid vessel under physiological pressure gradient
}

\author{
Dima Abi-Abdallah $\dagger$, Agnès Drochon $\dagger$, Vincent Robin $\ddagger$, and Odette Fokapu $\dagger$ \\ † Biomechanics and Bioengineering, University of Technology of Compiègne, France \\ ‡ Laboratory of Applied Mathematics, University of Technology of Compiègne, France \\ (June 2007)
}

Blood flow in a steady magnetic field has been of great interest over the past years. Many researchers have examined the effects of magnetic fields on velocity profiles and arterial pressure, and major studies focused on steady or sinusoidal flows. In this paper we present a solution for pulsed magnetohydrodynamic blood flow with a somewhat realistic physiological pressure wave obtained using a windkessel lumped model. A pressure gradient is derived along a rigid vessel placed at the output of a compliant module which receives the ventricle outflow. Then, velocity profile and flow rate expressions are derived in the rigid vessel in the presence of a steady transverse magnetic field. As expected, results showed flow retardation and flattening. The adaptability of our solution approach allowed a comparison with previously addressed flow cases and calculations presented a good coherence with those well established solutions.

Keywords: Static magnetic field, magnetohydrodynamic interactions, Hall effect, windkessel, lumped model.

\section{Introduction}

The increase in exposure to high magnetic fields caused by the wide use of Magnetic Resonance Imaging (MRI) as a standard medical procedure, has raised a concern in the research community and constituted an incentive for studying the effects of magnetic fields on human physiology and its impact on patients health. Especially that, in striving to achieve higher resolution and greater spectral separation, the MRI scanners static magnetic fields keep augmenting. Studies evaluating the effect of human or animal exposure to magnetic fields have shown no major changes, except for an increase of systolic blood pressure as well as alterations of the electrocardiogram (ECG) signal manifested as elevations of the $\mathrm{T}$ wave, all of which are due to blood flow.

The movement of a conducting fluid, such as the blood, in an externally applied

Corresponding author. Email: dima.abi-abdallah@u-psud.fr 
magnetic field is governed by the laws of magnetohydrodynamics. When the body is subjected to a magnetic field the charged particles of the blood flowing transversally to the field get deflected by the Lorentz force thus inducing electrical currents and voltages, across the vessel walls and in the surrounding tissues, strong enough to be detected at the surface of the thorax in the ECG. Furthermore, the interactions between these induced currents and the applied magnetic field can cause a reduction of flow rate and thus a reactive compensatory increase in blood pressure in order to retain a constant volume flow rate.

Magnetic field interactions with blood flow have been demonstrated by multiple authors throughout in vitro experiments $[1,2]$ where pressure and flow rate were measured, as well as in vivo studies such as $[3,4]$ where animal ECG alterations have been observed, and [5] where the effects on human vital signs were found to consist essentially in an arterial pressure increase.

Theoretical magnetohydrodynamic blood flow calculations have, however, been addressed much earlier and go back as far as the early sixties. Korchevskii and Marochnik [6] first proposed a velocity profile solution for blood flow between two parallel plates under a constant pressure gradient with a perpendicular magnetic field, under the assumption that blood is newtonian. Later other studies focused on flow in a rigid circular tube with non conducting walls placed in a transverse magnetic field to offer a more realistic model for blood flow in vessels. In this case, the most complete solution of the magnetohydrodynamic equations of a conducting fluid was proposed by Gold [7]. Setting a constant pressure gradient, Gold derived expressions for the velocity profile as well as induced fields and voltages. Vardanyan [8] subsequently published an approximate steady solution where velocity profile and flow rate were calculated by neglecting the induced fields. More recent studies were essentially based on these founding works, such as Keltner et al. [1] where a comparison was established between the results of Gold and Vardanyan to assess the consequences of neglecting the inductions. With the same hypothesis as Vardanyan, Sud et al. [9] later dealt with a sinusoidal pressure gradient that modeled a bit closer the pulsed nature of blood flow in arteries. The hypothesis of conducting walls was not introduced until Kinouchi et al. [10] who included inductions in the vessel and the surrounding tissues in the steady flow case in order to evaluate the induced ECG superimposed voltages.

In this work, we revisit the flow of blood as a newtonian fluid, in a circular rigid vessel, with non conducting walls, in the presence of a transverse constant magnetic field. Nevertheless, instead of taking a constant pressure gradient or a sinusoidal one, we apply a realistic pulsed pressure gradient derived using a windkessel lumped model, where the compliant module provides the input flow into the rigid vessel. Then, neglecting induced fields, we solve the magnetohydrodynamic equations to obtain velocity profile and flow rate expressions. 
The fact that our resolution method is based on Fourier decomposition makes the solutions easily adaptable to steady or sinusoidal cases, thus allowing a comparison with the previous well established studies.

\section{General equations and solution}

The flow of a conducting incompressible newtonian fluid in the presence of a magnetic field is defined by a combination of Maxwell's equations on one hand, and the Navier-Stokes equation including the magnetic force on the other, along with the conservation equation, as well as Ohm's law.

If we neglect the induced fields, the velocity profile can be solely defined by the Navier-Stokes equation, where the magnetic force term is evaluated using Ohm's law,

$$
\rho\left(\frac{\partial \vec{u}}{\partial t}+(\vec{u} \cdot \vec{\nabla}) \vec{u}\right)=-\vec{\nabla} p+\eta \Delta \vec{u}+\sigma(\vec{u} \wedge \vec{B}) \wedge \vec{B}
$$

where $\vec{B}$ is the magnetic field, $\vec{u}, \rho, \eta, \sigma$ are respectively the fluid velocity, density, viscosity and conductivity and $\vec{\nabla} p$ is the pressure gradient.

By assuming that the flow is unidirectional, axisymmetric with no swirl in a

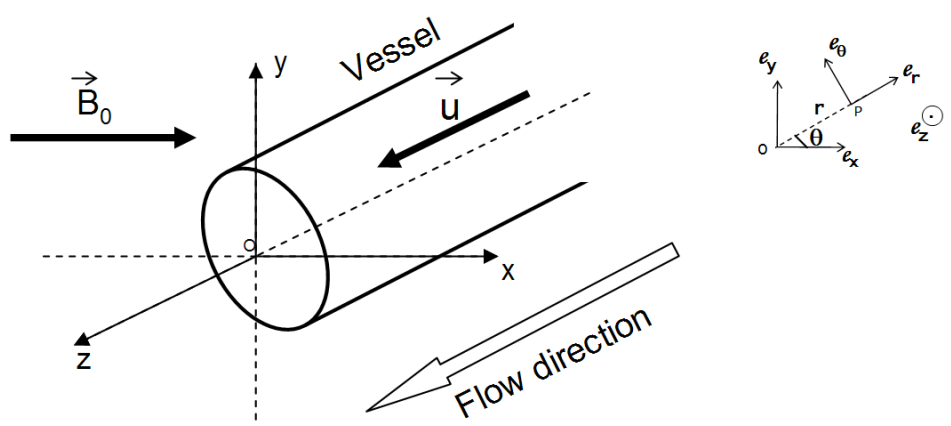

Figure 1. Flow model geometry

The vessel is represented by a cylindrical conduct where blood flows along the Oz axis, in the presence of a transverse steady magnetic field oriented in the Ox direction.

circular rigid vessel, its velocity can be written as $\vec{u}=(0,0, u(\tilde{r}, t))$ (fig. 1$)$. The fluid pressure is thus a function of the position $\mathrm{z}$ and time. The external constant magnetic field is applied transversally such as $\vec{B}=\left(B_{0} \cos \theta,-B_{0} \sin \theta, 0\right)$ and the vessel is considered to have non conducting walls. The flow would therefore be governed by the $O z$ projection of (1) which expressed in cylindrical coordinates gives,

$$
\frac{a^{2}}{\nu} \frac{\partial u(\tilde{r}, t)}{\partial t}=g(t)+\frac{\partial^{2} u(\tilde{r}, t)}{\partial \tilde{r}^{2}}+\frac{1}{\tilde{r}} \frac{\partial u(\tilde{r}, t)}{\partial \tilde{r}}-H_{a}^{2} u(\tilde{r}, t),
$$


with $\tilde{r}=\frac{r}{a}$, where $a$ represents the vessel radius, $H_{a}=B_{0} a \sqrt{\frac{\sigma}{\eta}}$ is the Hartmann number, $\nu=\frac{\eta}{\rho}$ is the kinematic viscosity, and

$$
g(t)=-\frac{a^{2}}{\eta} \frac{\partial p(t, z)}{\partial z}
$$

with boundary condition at the walls $u(1, t)=0$.

The proposed resolution method consists of a Fourier decomposition, followed by a Hankel transform.

Fourier series decomposition. The pulsed flow studied here is periodic with period $\mathrm{T}$, inverse of the cardiac frequency. $u(\tilde{r}, t)$ and $g(t)$ are thus $\mathrm{T}$ periodic time functions that can be decomposed in Fourier series such as,

$$
\begin{aligned}
u(\tilde{r}, t) & =\sum_{k=-\infty}^{+\infty} u_{k}(\tilde{r}) e^{i \omega_{k} t} & & \text { where: } u_{k}(\tilde{r})=\frac{1}{T} \int_{0}^{T} u(\tilde{r}, t) e^{-i \omega_{k} t} d t \\
g(t) & =\sum_{k=-\infty}^{+\infty} g_{k} e^{i \omega_{k} t} & & \text { where: } g_{k}=\frac{1}{T} \int_{0}^{T} g(t) e^{-i \omega_{k} t} d t
\end{aligned}
$$

with: $\omega_{k}=k \frac{2 \pi}{T}$.

By replacing in (2) we get,

$$
\begin{aligned}
\frac{a^{2}}{\nu} \sum_{k=-\infty}^{+\infty} i \omega_{k} u_{k}(\tilde{r}) e^{i \omega_{k} t}= & \sum_{k=-\infty}^{+\infty} g_{k} e^{i \omega_{k} t}+\sum_{k=-\infty}^{+\infty}\left(\frac{\partial^{2} u_{k}(\tilde{r})}{\partial \tilde{r}^{2}}+\frac{1}{\tilde{r}} \frac{\partial u_{k}(\tilde{r})}{\partial \tilde{r}}\right) e^{i \omega_{k} t} \\
& -H_{a}^{2} \sum_{k=-\infty}^{+\infty} u_{k}(\tilde{r}) e^{i \omega_{k} t} \\
\Leftrightarrow \frac{a^{2}}{\nu} i \omega_{k} u_{k}(\tilde{r}) & =g_{k}+\Delta u_{k}(\tilde{r})-H_{a}^{2} u_{k}(\tilde{r}) \quad \forall k \in \mathbb{Z} .
\end{aligned}
$$

Hankel Transform. For a function $f(r)$ defined over [0,1], the zero order Hankel transform is defined [11] as,

$$
H(f)=\left(f_{n}^{*}\right)_{n \in \mathbb{Z}} \quad ; \quad f_{n}^{*}=\int_{0}^{1} r f(r) J_{0}\left(r \lambda_{n}\right) d r \quad ;
$$

with the following properties,

$$
H(\Delta f(r))=-\lambda_{n}^{2} f_{n}^{*} \quad(\text { if } f(1)=0) \quad \text { and } \quad H\left(c_{\text {ste }}\right)=\frac{c_{\text {ste }}}{\lambda_{n}} J_{1}\left(\lambda_{n}\right) \quad,
$$


where $\lambda_{n}$ are the roots of Bessel function $J_{0}(x)$.

Knowing that $u(1, t)=0 \Leftrightarrow u_{k}(1)=0 \quad \forall k \in \mathbb{Z}$, applying the Hankel transform on equation (4) yields,

$$
u_{k, n}^{*}=\frac{J_{1}\left(\lambda_{n}\right)}{\lambda_{n}\left(i \omega_{k} \frac{a^{2}}{\nu}+\lambda_{n}^{2}+H_{a}^{2}\right)} g_{k} .
$$

Solution. To derive the solution we must inverse the Hankel transform to get the velocity's Fourier coefficients and then establish the velocity profile expression.

We know that if $H(f)=f_{n}^{*}$, then the inverse transform gives $f(r)$ such as, $f(r)=2 \sum_{n=1}^{\infty} \frac{J_{0}\left(\lambda_{n} r\right)}{J_{1}\left(\lambda_{n}\right)^{2}} f_{n}^{*}$.

From (5) we derive,

$$
u_{k}=2 \sum_{n=1}^{\infty} \frac{J_{0}\left(\lambda_{n} \tilde{r}\right)}{\lambda_{n} J_{1}\left(\lambda_{n}\right)} \frac{g_{k}}{i \omega_{k} \frac{a^{2}}{\nu}+\lambda_{n}^{2}+H_{a}^{2}},
$$

and therefore a velocity profile,

$$
u(\tilde{r}, t)=2 \sum_{k=-\infty}^{\infty} \sum_{n=1}^{\infty} \frac{J_{0}\left(\lambda_{n} \tilde{r}\right)}{\lambda_{n} J_{1}\left(\lambda_{n}\right)} \quad \frac{g_{k}}{i \omega_{k} \frac{a^{2}}{\nu}+\lambda_{n}^{2}+H_{a}^{2}} e^{i \omega_{k} t} .
$$

The flow rate will then be given as,

$$
\begin{aligned}
q(t) & =\iint_{A} u d A=\int_{0}^{2 \pi} \int_{0}^{a} u(r, t) r d r d \theta \\
& =4 \pi a^{2} \sum_{k=-\infty}^{\infty} \sum_{n=1}^{\infty} \frac{e^{i \omega_{k} t}}{\lambda_{n} J_{1}\left(\lambda_{n}\right)} \frac{g_{k}}{i \omega_{k} \frac{a^{2}}{\nu}+\lambda_{n}^{2}+H_{a}^{2}} \int_{0}^{1} J_{0}\left(\lambda_{n} \tilde{r}\right) \tilde{r} d \tilde{r}
\end{aligned}
$$

Using the fact that $\int x^{n} J_{n-1}(x) d x=x^{n} J_{n}(x)$ ( [12], p.137), we get,

$$
q(t)=4 \pi a^{2} \sum_{k=-\infty}^{\infty} \sum_{n=1}^{\infty} \frac{e^{i \omega_{k} t}}{\lambda_{n}^{2}} \frac{g_{k}}{i \omega_{k} \frac{a^{2}}{\nu}+\lambda_{n}^{2}+H_{a}^{2}} .
$$

\section{Pressure gradient expression}

In the literature, studies concerning pulsed flows in rigid tubes usually consider sinusoidal pressure gradients [11] and [9]. In this work, in order to solve the velocity profile, we seek to impose a realistic physiological pressure gradient. For this, we use the 3-element Windkessel lumped model where a compliant module represents the large arteries and a pure resistance represents the peripheral rigid vessels. We then derive a pressure gradient, governed by the compliant module, to be applied on a rigid vessel beyond the large arteries.

Lumped models are often used to represent blood flow in the arterial system. 


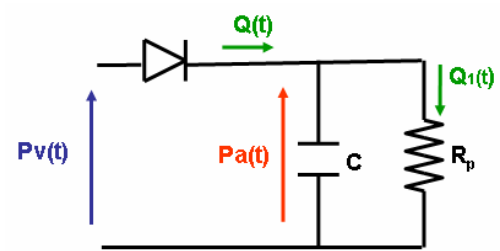

(a) 2-element Windkessel

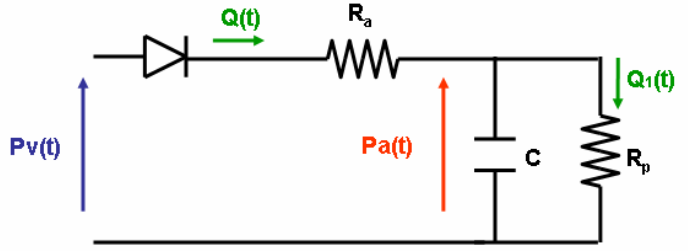

(b) 3-element Windkessel

Figure 2. Windkessel lumped models

$P v(t)$ :left ventricular pressure, $P a(t)$ :aortic pressure, $Q(t)$ :output flow rate of the left ventricle, $Q_{1}(t)$ :input flow rate to the peripheral vessels, $R_{a}$ :resistance of the aorta and the large arteries,

$C$ :compliance of the aorta and large arteries, $R_{p}$ :total peripheral resistance of small arteries, arterioles and capillaries

They rely on an analogy with electric circuits where currents represent arterial blood flows and voltages represent arterial pressures. In such models, resistances stand for resistance to flow (arterial and peripheral) resulting from viscous dissipation inside the vessels, capacitors represent volume compliance of the vessels, and inductors represent blood inertia.

In the circulatory system, the small caliber arteries can be considered as rigid. These arteries get at their input a flow rate imposed by the large compliant arteries and can be modeled using pure resistances [13].

In the following section we will establish a model that will allow us to evaluate this flow rate and therefore deduce an expression of pressure gradient along a rigid vessel.

The Windkessel model, conceived by Otto Frank in 1899 and inspired by air chamber pumps used in fire engines, is a quite simple configuration that describes the flow at the heart output and into the systemic arteries. The model consists of an elastic accumulation chamber placed in a rigid conduct preceded by a valve and followed by a Poiseuille hydraulic resistance. When the valve is open (systole) part of the ventricular flow accumulates in the chamber, and the rest flows in the resistance. When the valve is closed (diastole) the blood which had accumulated in the chamber is forced out through the resistance. This electrical model was initially conceived with two elements comprising a capacitor that represents the elasticity of large arteries and a peripheral resistance that stands for the resistance of small arteries and arterioles (fig.2(a)). The input pressure of the circuit is the left ventricular pressure, assuming a null pressure at the vena cava. A diode placed at the circuit's entry plays the role of the aortic valve which lets the flow get through only when the ventricular pressure is superior to that of the aorta. This 2-element model was later transformed into a 3-element model (fig.2(b)) where an additional resistance was introduced to take into account the resistance of the aorta and large arteries [14] . The latter model was found to produce quite realistic pressure and flow rate curves that correctly reproduce experimental data [15] and thus remains very widely used to this date. 
In the following section we adopt the 3-element windkessel model and compute an expression for pressure along a pure resistance at the output of the compliant module. We first define a mathematical expression to model real ventricular pressure input such as [15],

$$
P v(t)= \begin{cases}\frac{P_{\max }}{2}(1-\cos 2 \gamma t) & 0 \leq t \leq t_{p} \\ 0 & t_{p} \leq t \leq T\end{cases}
$$

with $\gamma=\frac{\pi}{t_{p}}$.

The differential equation defining the 3 -element circuit can be written as,

$$
\frac{d P a}{d t}+\frac{P a}{\tau}=\frac{Q(t)}{C}
$$

where $\tau=C R_{p}$.

Solving for $P a(t)$ in each cardiac cycle phase : diastole and isovolumetric contraction phases where $Q(t)=0$, as well as the ejection phase where $Q(t)=\frac{P v(t)-P a(t)}{R_{a}}$, while ensuring curve continuity between the phases yields the aortic pressure expression,

$$
P a(t)=\left\{\begin{array}{lll}
P_{s} e^{-\frac{t+T-t_{s}}{\tau}} & 0 \leq t \leq t_{1} \quad \text { (isovolumetric contraction) } \\
K e^{-\frac{t-t_{1}}{Z \tau}}+A(t) & t_{1} \leq t \leq t_{s} & \text { (ejection) } \\
P_{s} e^{-\frac{t-t_{s}}{\tau}} & t_{s} \leq t \leq T & \text { (diastole) }
\end{array}\right.
$$

with,

$$
\begin{gathered}
A(t)=\frac{R_{p}}{R_{a}+R_{p}} \frac{P_{\max }}{2}\left(1-\frac{2 \gamma Z \tau \sin 2 \gamma t+\cos 2 \gamma t}{1+4 \gamma^{2} Z^{2} \tau^{2}}\right) \\
P_{s}=P v\left(t_{s}\right) \quad ; \quad K=P v\left(t_{s}\right) e^{-\frac{t_{1}+T-t_{s}}{\tau}}-A\left(t_{1}\right) \quad ; \quad Z=\frac{R_{a}}{R_{a}+R_{p}} ;
\end{gathered}
$$

$t_{1}$ denotes the beginning of the ejection phase when $P a$ becomes less than $P v$, $t_{s}$ denotes the end of systole when $P a$ becomes greater than $P v$, and $t_{p}$ is the instant at which the pressure in the ventricle drops to zero, and $T$ is the cardiac cycle period.

The flow rate in the peripheral resistance is given by $Q_{1}(t)=\frac{P a(t)}{R_{p}}$ and the pressure drop along a peripheral vessel of radius $a$ and length $L$ would be obtained by multiplying the flow rate with a hydraulic Poiseuille resistance $\frac{8 \eta L}{\pi a^{4}}$ yielding $\frac{d P(t)}{L}=-\frac{8 \eta}{\pi a^{4}} Q_{1}(t)$, and thus,

$$
-\frac{\partial p(t, z)}{\partial z}=\frac{8 \eta}{\pi a^{4}} \begin{cases}\frac{P_{s}}{R_{p}} e^{-\frac{t+T-t_{s}}{\tau}} & 0 \leq t \leq t_{1} \\ \frac{K}{R_{p}} e^{-\frac{t-t_{1}}{Z \tau}}+\frac{A(t)}{R_{p}} & t_{1} \leq t \leq t_{s} \\ \frac{P_{s}}{R_{p}} e^{-\frac{t-t_{s}}{\tau}} & t_{s} \leq t \leq T\end{cases}
$$


In order to derive the velocity profile expression by (7) we need to compute the Fourier coefficients $g_{k}$ of $g(t)$ defined in (3). After Fourier integral calculation for each of the three phases,

$$
\begin{aligned}
g_{k}= & \frac{1}{T} \int_{0}^{T}-\frac{a^{2}}{\eta} \frac{\partial p(t, z)}{\partial z} e^{-i \omega_{k} t} d t \\
= & \frac{8}{T \pi a^{2} R_{p}}\left(\int_{0}^{t_{1}} P_{s} e^{-\frac{t+T-t_{s}}{\tau}} e^{-i \omega_{k} t} d t+\int_{t_{1}}^{t_{s}}\left(K e^{-\frac{t-t_{1}}{Z \tau}}+A(t)\right) e^{-i \omega_{k} t} d t\right. \\
& \left.+\int_{t_{s}}^{T} P_{s} e^{-\frac{t-t_{s}}{\tau}} e^{-i \omega_{k} t} d t\right),
\end{aligned}
$$

we get,

$$
\begin{aligned}
g_{k}= & \frac{8}{T \pi a^{2} R_{p}}\left\{\frac{P_{s}}{\frac{1}{\tau}+i \omega_{k}} e^{-\frac{T-t_{s}}{\tau}}\left(1-e^{-t_{1}\left(\frac{1}{\tau}+i \omega_{k}\right)}-e^{-i \omega_{k} T}+e^{-t_{s}\left(\frac{1}{\tau}+i \omega_{k}\right)+\frac{T}{\tau}}\right)\right. \\
& +\frac{K}{\frac{1}{Z \tau}+i \omega_{k}} e^{\frac{t_{1}}{Z \tau}}\left(e^{-t_{1}\left(\frac{1}{Z \tau}+i \omega_{k}\right)}-e^{-t_{s}\left(\frac{1}{Z \tau}+i \omega_{k}\right)}\right) \\
& \left.+\frac{R_{p}}{R_{a}+R_{p}} \frac{P_{\max }}{2}\left[f_{k}-\left.\frac{e^{-i \omega_{k} t}\left[\left(-i \omega_{k}-2 \gamma \epsilon\right) \cos (2 \gamma t)+\left(2 \gamma-i \omega_{k} \epsilon\right) \sin (2 \gamma t)\right]}{\left(1+4 \gamma^{2} Z^{2} \tau^{2}\right)\left(4 \gamma^{2}-\omega_{k}^{2}\right)}\right|_{t_{1}} ^{t_{s}}\right]\right\},
\end{aligned}
$$

where $\epsilon=2 \gamma Z \tau$ and $f_{k}=\left\{\begin{array}{ll}t_{s}-t_{1} & k=0 \\ \frac{e^{-i \omega_{k} t_{1}}-e^{-i \omega_{k} t_{s}}}{i \omega_{k}} & k \neq 0\end{array}\right.$.

\section{Results}

All numerical computations were done using the parameter values shown in table 1 .

Figure 3 shows the pressure and flow rate curves computed using the 3 -element Windkessel model (fig.2(b)). They agree very well with measured pressure curves shown in [16]. From these computed results we can calculate other cardiac cycle parameters. We find a mean cardiac output of $77 \mathrm{~cm}^{3} / \mathrm{s}(4.62 \mathrm{l} / \mathrm{min})$, a stroke volume of $62 \mathrm{~cm}^{3}$ and a systole duration of $t_{s}=30 \% \mathrm{~T}$, all coinciding well with the normal range values (Comolet [17], for example, gives C.O. $\approx 87 \mathrm{~cm}^{3} / \mathrm{s}, S . V . \approx 70 \mathrm{~cm}^{3}$ and $t_{s} \approx 37 \% \mathrm{~T}$ for $P_{\max }=140 \mathrm{mmHg}$ ).

Figure 4(a) shows the pressure gradient wave in the rigid vessel with radius $a$, as well as the flow rates for different Hartmann numbers. It could be noted that for small $H_{a}$ the variation dynamics of the flow rate (and velocity) is a lot slower than that of the pressure gradient, however for larger $H_{a}$ the flow rate 
Table 1. Numerical values

\begin{tabular}{|c|r|r|}
\hline \multicolumn{4}{|c|}{ Windkessel circuit components } \\
\hline$R_{a}$ & 0.0334 & $\mathrm{mmHg} . \mathrm{s} / \mathrm{cm}^{3}$ \\
\hline$R_{p}$ & 1 & $\mathrm{mmHg} . \mathrm{s} / \mathrm{cm}^{3}$ \\
\hline$C$ & 0.77 & $\mathrm{~cm}^{3} / \mathrm{mmHg}$ \\
\hline \multicolumn{4}{|c|}{ Blood characteristics } \\
\hline$\eta$ & $4.10^{-3}$ & $\mathrm{Pa.s}$ \\
\hline$\rho$ & 1050 & $\mathrm{~kg} / \mathrm{m}^{3}$ \\
\hline$\sigma$ & 0.5 & $\mathrm{~m} / \mathrm{m}^{-3}$ \\
\hline \multicolumn{4}{|c|}{ Vessel radius } \\
\hline$a$ & 0.3 & $\mathrm{~cm}$ \\
\hline \multicolumn{4}{|c|}{ Cardiac cycle parameters } \\
\hline$f r e q$ & \multicolumn{3}{c}{75} & $\mathrm{mHg}$ \\
\hline$P_{\max }$ & 120 & $\mathrm{mpm}$ \\
\hline$t_{p}$ & $50 \% T$ & \\
\hline
\end{tabular}

The resistance and capacitor values are chosen based on [15] and agreeing with [14], blood characteristics are taken from [10] and the cardiac cycle parameters are chosen to match the average typical values.
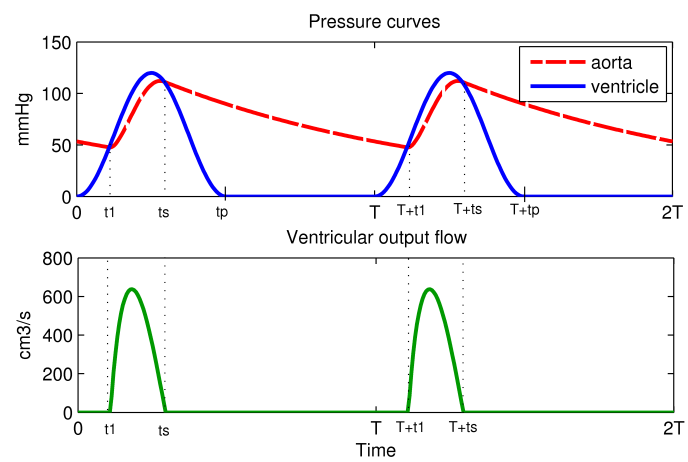

Figure 3. Ventricular pressure wave and aortic pressure and flow rate curves

Pressure and flow rate waves computed using the windkessel model over two cardiac cycles at 75

bpm. The ventricular pressure $P v(t)$ is set as in equation (9), the aortic pressure $P a(t)$ is computed by (11), and the aortic flow rate (ventricle output) is $Q(t)=\frac{P v(t)-P a(t)}{R_{a}}$

curves tends to follow that of the pressure. The retardation of the movement can also be clearly depicted, as the flow slows down when the magnetic field intensity increases(fig.4(b)).

Note that by applying the Poiseuille standard formula for stationary flows $q_{p o i s}=\frac{\pi a^{4}}{8 \eta} \frac{\Delta p}{\Delta z}$, in a vessel of the same caliber under a pressure gradient equal to the mean value of the computed pulsed gradient, we would get approximatly $77 \mathrm{~cm}^{3} / \mathrm{s}$. Which is the value we get by computing the mean value of the pulsed flow rate for $H_{a}=0$.

Given that a Poiseuille profile yields a maximum velocity $U_{\max }=\frac{a^{2}}{4 \eta} \frac{\Delta p}{\Delta z} \approx$ $544 \mathrm{~cm} / \mathrm{s}$, figures $5(\mathrm{a})$ and $5(\mathrm{~b})$ represent normalized velocity profiles as ratios to this value for $H_{a}=0$ and $H_{a}=2$ respectively. 

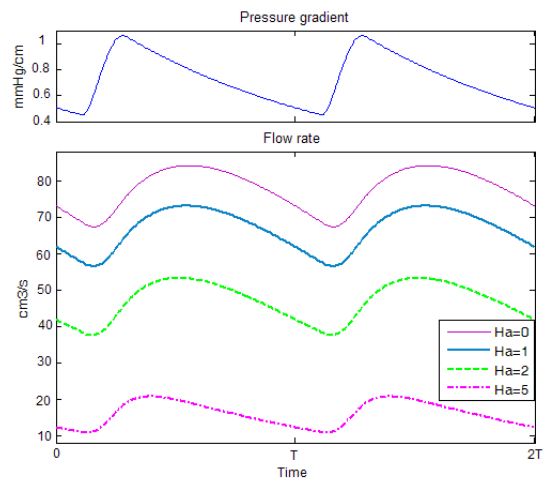

(a) Pressure gradient and flow rate in a rigid vessel: The pressure gradient is calculated by (12), and the flow rate by (8) for different Hartmann numbers for a pulsed flow in a rigid vessel which characteristic values are given in table 1

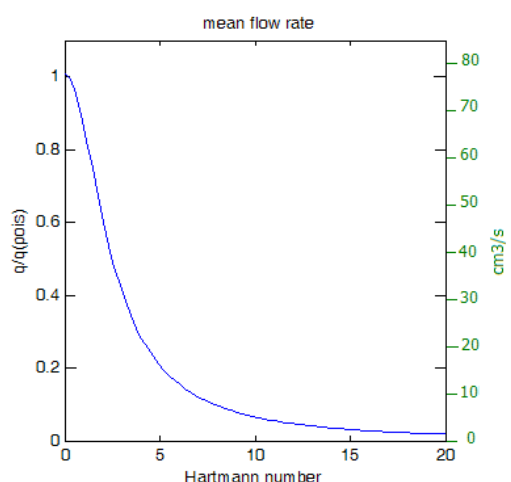

(b) Mean flow rate as function of the Hartman number: The graduations on the left represent a flow rate normalized as a ratio to a Poiseuille flow rate, while the graduations on the right represent a flow rate in $\mathrm{cm}^{3} / \mathrm{s}$

Figure 4. Flow rate for various field intensities

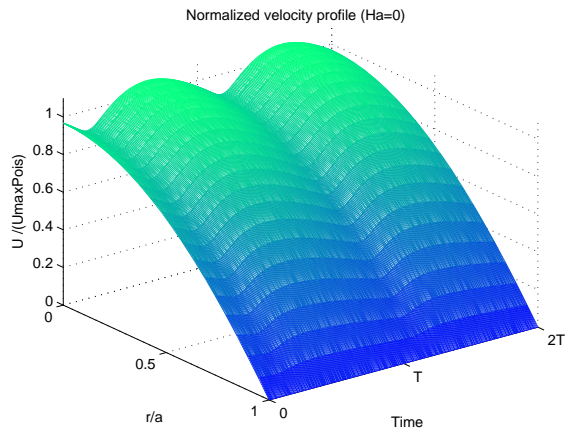

(a) $\mathrm{Ha}=0$

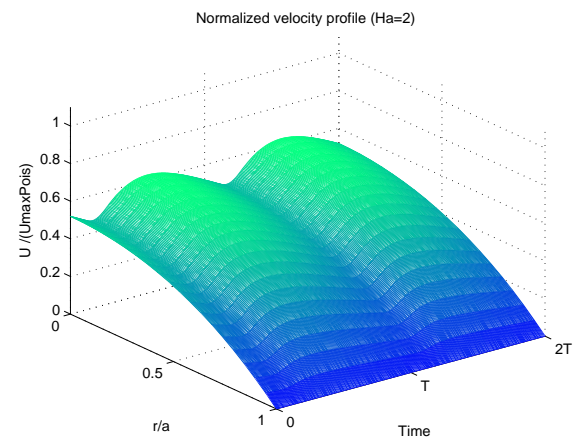

(b) $\mathrm{Ha}=2$

Figure 5. Normalized velocity profiles

The velocity is computed from (7) and the normalization is done respectively to the Poiseuille

$$
U_{\max } \approx 544 \mathrm{~cm} / \mathrm{s}
$$

\section{Comparison with other flow types}

In this section we discuss the obtained results by comparing them to other well established cases of blood flows, whether stationary or pulsed, in the presence of a magnetic field or not, with or without induced fields. 


\subsection{Stationary flows}

One of the advantages of our resolution method using the Fourier series is that we can compare the results of our pulsed flow with a stationary one, simply by limiting the calculations to the first harmonic $k=0$. The pressure gradient can then be expressed as $-\frac{\partial p}{\partial z}=\frac{\eta}{a^{2}} g_{0}$, the velocity profile and flow rate would be given by,

$$
\begin{aligned}
u_{0}(\tilde{r}) & =\frac{g_{0}}{H_{a}^{2}}\left(1-\frac{I_{0}\left(H_{a} \tilde{r}\right)}{I_{0}\left(H_{a}\right)}\right), \\
q_{0} & =2 \pi a^{2} \frac{g_{0}}{H_{a}^{2}}\left(\frac{1}{2}-\frac{I_{1}\left(H_{a}\right)}{H_{a} I_{0}\left(H_{a}\right)}\right),
\end{aligned}
$$

where $I_{0}$ and $I_{1}$ are modified Bessel functions, and $g_{0}$ is the mean value of $g(t)$ : $g_{0}=\frac{1}{T} \int_{0}^{T} g(t) d t$, which expression can be calculated from (13) for $\omega_{k}=0$.

With no magnetic field (Poiseuille flow). The stationary well known Poiseuille flow in a rigid vessel is given by $u_{\text {pois }}(\tilde{r})=-\frac{a^{2}}{4 \eta} \frac{\Delta p}{\Delta z}\left(1-\tilde{r}^{2}\right)$.

By applying the same pressure gradient as described above, the velocity profile can be expressed as,

$$
u_{\text {pois }}(\tilde{r})=\frac{g_{0}}{4}\left(1-\tilde{r}^{2}\right) .
$$

If we expand (14) for small values of $H_{a}$, while knowing that, $I_{0}(x)=\sum_{k=0}^{\infty} \frac{\left(\frac{1}{4} x^{2}\right)^{k}}{k !^{2}}([18]$ p. 375$)$, we get that,

$$
\lim _{H_{a} \rightarrow 0} u_{0}(\tilde{r})=u_{\text {pois }}(\tilde{r})
$$

Figure 6(a) compares non dimensional flow rates $\left(2 u_{0} / g_{0}\right)$ for various field intensities to a Poiseuille flow $\left(2 u_{\text {pois }} / g_{0}\right)$ in the absence of a magnetic field. It clearly shows the influence of a magnetic field which is manifested by a flow retardation and a flattening of the velocity profile, which is further accentuated as $H_{a}$ increases.

In the presence of a magnetic field neglecting induced fields. For $\mathrm{k}=0$ (4) can be written,

$$
\Delta u_{0}(\tilde{r})-H_{a}^{2} u_{0}(\tilde{r})=-g_{0} \quad .
$$

This is the same equation as established by Vardanyan [8] who studied the case of a steady flow in a magnetic field while neglecting the induced fields, and for which he derived the solution,

$$
u_{v a r d}(\tilde{r})=\frac{g_{0}}{H_{a}^{2}}\left(1-\frac{I_{0}\left(H_{a} \tilde{r}\right)}{I_{0}\left(H_{a}\right)}\right) .
$$


This matches exactly with (14). Furthermore, if we compare it to the expression in (7) for $\mathrm{k}=0$, we deduce that we must have,

$$
2 \sum_{n=1}^{\infty} \frac{J_{0}\left(\lambda_{n} \tilde{r}\right)}{\lambda_{n} J_{1}\left(\lambda_{n}\right)} \quad \frac{1}{\lambda_{n}^{2}+H_{a}^{2}}=\frac{1}{H_{a}^{2}}\left(1-\frac{I_{0}\left(H_{a} \tilde{r}\right)}{I_{0}\left(H_{a}\right)}\right),
$$

which we in fact find if we numerically compute the sum.

In the presence of a magnetic field with induced fields. Gold [7] established an exact solution for stationary blood flow in a magnetic field taking into consideration induced current and magnetic field,

$$
\begin{aligned}
u_{\text {gold }}(\tilde{r}, \theta) & =\frac{g_{0}}{2 H_{a}}\left[e^{-\frac{H_{a}}{2} r \cos \theta} \sum_{n=0}^{\infty} \epsilon_{n} \frac{I_{n}^{\prime}\left(\frac{H_{a}}{2}\right)}{I_{n}\left(\frac{H_{a}}{2}\right)} I_{n}\left(\frac{H_{a}}{2} r\right) \cos n \theta\right. \\
& \left.+e^{\frac{H_{a}}{2} r \cos \theta} \sum_{n=0}^{\infty}(-1)^{n} \epsilon_{n} \frac{I_{n}^{\prime}\left(\frac{H_{a}}{2}\right)}{I_{n}\left(\frac{H_{a}}{2}\right)} I_{n}\left(\frac{H_{a}}{2} r\right) \cos n \theta\right],
\end{aligned}
$$

where,

$$
\epsilon_{n}=\left\{\begin{array}{l}
1 \text { pour } n=0 \\
2 \text { pour } n>0
\end{array}\right.
$$

To compare this $\theta$ dependent velocity profile to our axially symmetric case, we take the mean velocity,

$$
\bar{u}_{\text {gold }}=g_{0} \sum_{n=0}^{\infty}(-1)^{n} \epsilon_{n} \frac{I_{n}^{\prime}\left(\frac{H_{a}}{2}\right)}{H_{a} I_{n}\left(\frac{H_{a}}{2}\right)}\left[\left(1+\frac{n^{2}}{\left(\frac{H_{a}}{2}\right)^{2}}\right) I_{n}^{2}\left(\frac{H_{a}}{2}\right)-I_{n}^{\prime 2}\left(\frac{H_{a}}{2}\right)\right],
$$

and compare it to

$$
\bar{u}_{0}=2 \frac{g_{0}}{H_{a}^{2}}\left(\frac{1}{2}-\frac{I_{1}\left(H_{a}\right)}{H_{a} I_{0}\left(H_{a}\right)}\right),
$$

computed from (14) by averaging over the cross sectional area.

In accordance with what was demonstrated by Keltner et al. [1], we find that neglecting induced fields leads to an overestimation of the retardation and flattening effects, especially for high intensity fields (fig. 6(b)).

\subsection{Periodic flows}

Studies on periodic flows generally consider sine shaped pressure gradients. In order to compare results found in the literature to ours it suffices to restrain our pressure gradient to its first harmonic. 


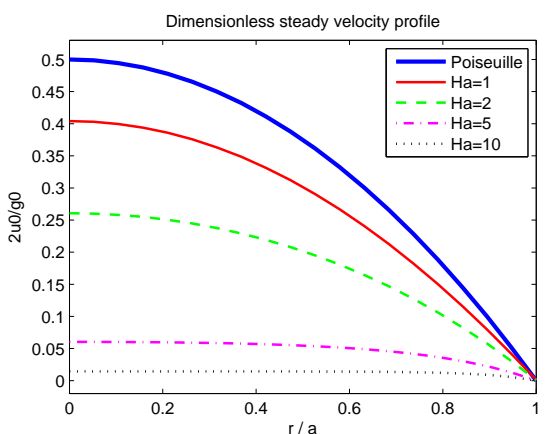

(a) Non dimensional velocity profiles computed from (14) for various values of $H_{a}$ and compared to the Poiseuille $(16)$ flow $\left(B_{0}=0\right)$

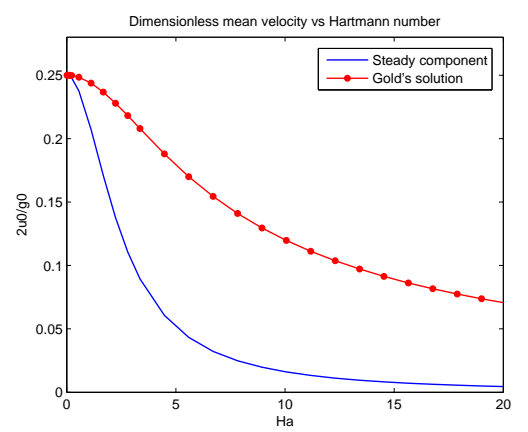

(b) Comparison between the mean velocity computed from (20) and Gold's exact solution (19)

Figure 6. Comparison to other steady flows

In the absence of any magnetic field (Womersley flow). The sinusoidal flow in a rigid conduct has been studied by many authors, but the most significant results were established by Womersley [19]. For a pressure gradient such as,

$$
-\frac{1}{\rho} \frac{\partial p(t)}{\partial z}=a_{0}+a_{1} e^{i \omega t} .
$$

Womersley solved a velocity profile given by

$$
u_{w o m}(\tilde{r}, t)=\frac{a_{0} a^{2}}{4 \nu}\left(1-\tilde{r}^{2}\right)+\frac{a_{1} a^{2}}{i \nu \alpha^{2}}\left[1-\frac{J_{0}\left(i^{3 / 2} \alpha \tilde{r}\right)}{J_{0}\left(i^{3 / 2} \alpha\right)}\right] e^{i \omega t},
$$

where $\alpha=a \sqrt{\frac{\omega}{\nu}}$ is the Witzig-Womersley number.

In our calculations, the pressure gradient corresponding to the first harmonic along with the continuous component can be written as $-\frac{1}{\rho} \frac{\partial p}{\partial z}=$ $\frac{\nu}{a^{2}}\left(g_{0}+g_{1} e^{i \omega t}\right)$. In order to compare our results with those of Womersley, we must set $a_{0}=\frac{\nu}{a^{2}} g_{0}$ and $a_{1}=\frac{\nu}{a^{2}} g_{1}$.

Equation (22) can thus be written as,

$$
u_{w o m}(\tilde{r}, t)=\frac{g_{0}}{4}\left(1-\tilde{r}^{2}\right)+\frac{g_{1}}{i \alpha^{2}}\left[1-\frac{J_{0}\left(i^{3 / 2} \alpha \tilde{r}\right)}{J_{0}\left(i^{3 / 2} \alpha\right)}\right] e^{i \omega t},
$$

and the flow rate would be given by,

$$
q_{w o m}(t)=2 \pi a^{2}\left(\frac{g_{0}}{16}+\frac{g_{1}}{i \alpha^{2}}\left[\frac{1}{2}-\frac{J_{1}\left(i^{3 / 2} \alpha\right)}{i^{3 / 2} \alpha J_{0}\left(i^{3 / 2} \alpha\right)}\right] e^{i \omega t}\right) .
$$

Afterwards we compute the velocity and flow rate using (23) and (24) on one hand, and (7) and (8) on the other for $0 \leq k \leq 1$. We verify, in fact, that in the 


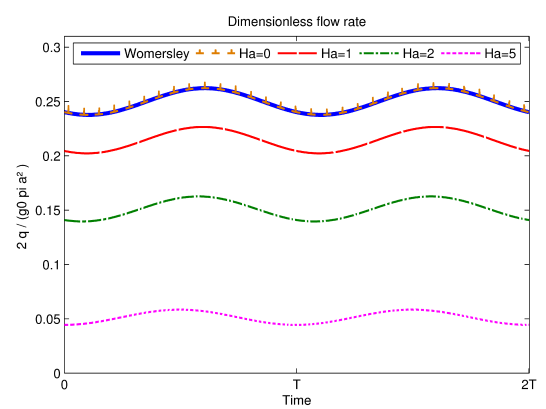

(a) Non dimensional flow rates $\frac{2 q}{g_{0} \pi a^{2}}$ with $q$ calculated by (8) for various $H_{a}$ compared to the Womersley flow where $q$ is given by (24)

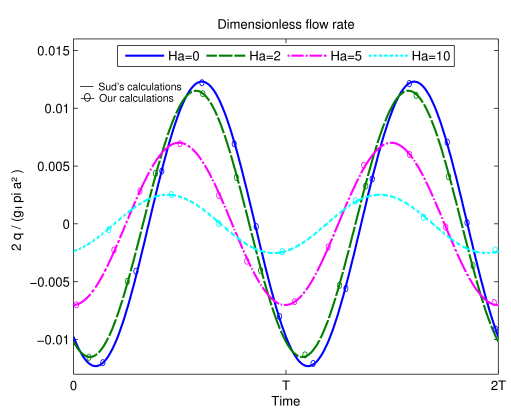

(b) Plots of $\frac{2 q}{g_{1} \pi a^{2}}$ where $q$ is calculated by (8) and compared to Sud's case where $q$ is given by (30) for various $H_{a}$

Figure 7. Comparison with other sinusoidal flows

absence of any magnetic field $\left(H_{a}=0\right)$ we get the same results as Womersley. Figure 7 (a) plots flow rates calculated for various $H_{a}$ as well as a flow rate curve obtained by Womersley's expression, with which a perfect match can be depicted for $H_{a}=0$.

In the presence of a magnetic field neglecting induced fields. Sud et al. [9] considered the case of a sinusoidal flow in a rigid conduct in the presence of a external transverse magnetic field while neglecting the inductions. They obtained non dimensional velocity profile such as,

$$
u_{s u d}^{*}\left(r^{*}, t^{*}\right)=\frac{A^{*}}{i+\frac{H_{a}^{2}}{R_{e}}}\left[1-\frac{J_{0}\left(\sqrt{-H_{a}^{2}-i R_{e}} r^{*}\right)}{J_{0}\left(\sqrt{-H_{a}^{2}-i R_{e}}\right)}\right] e^{i t^{*}},
$$

by imposing a pressure gradient given by,

$$
-\frac{\partial p^{*}}{\partial z^{*}}=A^{*} e^{i t^{*}}
$$

with,

$$
z^{*}=z / a, \quad r^{*}=r / a, \quad t^{*}=\omega t, \quad u^{*}=\frac{u}{\omega a}, \quad p^{*}=\frac{p}{\rho a^{2} \omega^{2}}, \quad A^{*}=\frac{A}{\rho \omega^{2} a}, \quad R_{e}=\frac{\omega a^{2}}{\nu} .
$$


Winding up to the dimensional form we get,

$$
\begin{aligned}
u_{\text {sud }}\left(r^{*}, t\right) & =\frac{A}{\rho\left(i \omega+\frac{H_{a}^{2} \nu}{a^{2}}\right)}\left[1-\frac{J_{0}\left(\sqrt{-H_{a}^{2}-i \frac{\omega a^{2}}{\nu}} r^{*}\right)}{J_{0}\left(\sqrt{-H_{a}^{2}-i \frac{\omega a^{2}}{\nu}}\right)}\right] e^{i \omega t}, \\
-\frac{\partial p}{\partial z} & =A e^{i \omega t} .
\end{aligned}
$$

In order to compare those results with our calculations we set $\frac{A}{\rho}=\frac{\nu}{a^{2}} g_{1}$ in (27) and $k=1$ in (7).

Using the Witzig-Womersley number $\alpha=a \sqrt{\frac{\omega}{\nu}}$ we obtain the following velocity and flow rate expressions,

$$
\begin{gathered}
u_{\text {sud }}\left(r^{*}, t\right)=\frac{g_{1}}{i \alpha^{2}+H_{a}^{2}}\left[1-\frac{J_{0}\left(\sqrt{-H_{a}^{2}-i \alpha^{2}} r^{*}\right)}{J_{0}\left(\sqrt{-H_{a}^{2}-i \alpha^{2}}\right)}\right] e^{i \omega t}, \\
q_{\text {sud }}(t)=\frac{2 \pi a^{2} g_{1}}{i \alpha^{2}+H_{a}^{2}}\left[\frac{1}{2}-\frac{J_{1}\left(\sqrt{-H_{a}^{2}-i \alpha^{2}}\right)}{\sqrt{-H_{a}^{2}-i \alpha^{2}} J_{0}\left(\sqrt{-H_{a}^{2}-i \alpha^{2}}\right)}\right] e^{i \omega t} .
\end{gathered}
$$

Note that for $H_{a}=0$ we get the same expressions as those of the Womersley's flow with no continuous component.

By calculating the velocities and flow rates using (29) and (30) we get matching results with calculations by (7) and (8). Figure 7(b) plots different flow rate curves computed using our expression as well as that of Sud for various $H_{a}$, the curves superimpose perfectly for the same field intensity.

In the presence of a magnetic field with induced fields. To the best of our knowledge, up to this date, no study has considered the case of a periodic blood flow in the presence of a magnetic field while taking into consideration induced fields.

\section{Discussion}

\subsection{On the use of the windkessel model}

The pulsed pressure gradient used in this work is derived from a lumped model. The major disadvantage of such models, is that they don't take into account the pressure wave propagation phenomenon. They behave as if the propagation were immediate. This however is of no inconvenience in the case 
of this study since we are interested in rigid vessel flows, where in fact the pressure propagation is very fast. If propagation were to be considered here it should be incorporated in the large arteries, however in our case this would have only introduced a certain delay and amplitude reduction in the rigid vessel. Hence a windkessel model offers a simple enough configuration without being very detrimental since we are not studying the entire system and not trying to simulate waves with exact values for a given vessel.

The used windkessel model might be further expanded to include four elements by adding an inductor to represent the blood inertia in the large arteries. This will ensure a better fit of real pressure waves [20]. It could also be enhanced by changing the output load and replacing $R_{p}$ with another windkessel model, thus introducing inductors and capacitors for small arteries. Nevertheless, it has been demonstrated that this would not greatly improve the pressure and flow rate curves observed at the top of the circulatory tree [15].

\subsection{On the choice of vessel model and flow conditions}

In this study, a realistic pressure gradient is derived along a rigid vessel placed at the output of a compliant module which receives the ventricle outflow. The compliant module includes the resistance and compliance of the aorta and large arteries. The vessel placed at the output of the elastic chamber had thus to be of a small caliber (just small enough to be considered as rigid [13], and big enough not to be too far down the circulatory tree). Since no bifurcations are taken into account, the rigid vessel receives all the aortic flow, and this leads to calculated flow rates (and velocities) that are much higher than the real flow rates that occur generally in arteries of $0.3 \mathrm{~cm}$ radius (for example, in the femoral artery which has a radius of $0.4 \mathrm{~cm}$, the blood velocity reaches a maximum of $100 \mathrm{~cm} / \mathrm{s}$ [15] only, whereas in our calculations, we obtained velocities reaching five times this physiological value). We could obtain reasonable rates by inserting parallel branches in the periphery, thus forming a tree of resistances where each resistance bifurcates in two, at each level of the tree. Then the flow would be cut in half at each bifurcation point, so that at the second level, for example, the flow would have already been reduced to one fourth its original value. With this rough approximation of the peripheral circulatory tree, all calculations would remain unchanged, then, depending on the level at which the vessel of interest is found the resulting values would be divided by $2^{\text {level }}$.

However, we should note that, during exposure to static magnetic fields it is the large arteries flows that are mostly affected by the magnetic interactions. In order to get an estimate of flows that might occur in a large vessel, we could artificially place a $1 \mathrm{~cm}$ radius vessel at the output of our compliant 


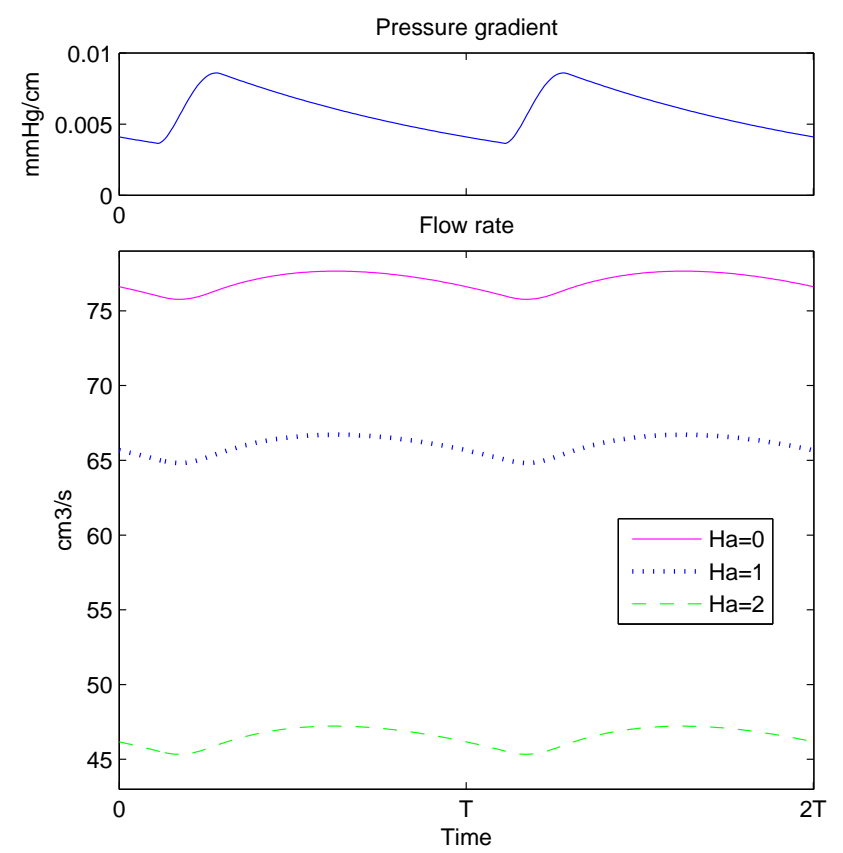

Figure 8. Pressure gradient and flow rate in a $1 \mathrm{~cm}$ radius rigid vessel

The pressure gradient is calculated by (12), and the flow rate by (8) for different Hartmann

numbers

module, and perform calculations (shown in fig. 8) similar to those presented in figure 4(a) (the curve corresponding to $\mathrm{Ha}=5$ is not shown in order to be able to enlarge the ordinate scale). When comparing both figures, we can see that the oscillations amplitudes are more dampened here. Note that the non-dimensional graph corresponding to the data of fig.8 would be the same as that of fig.4(b). Even though the considered vessel has the same caliber as the aorta, the pressure gradient and flow values obtained here would not reflect closely those observed in the aorta, since the aorta is not rigid but compliant and it receives it's flow directly from the ventricle and not through the modeled windkessel chamber.

Furthermore, because of the growing field of small animal imaging using MRI, it would also be interesting to assess to what extent MRI measurements can alter the haemodynamics in mice. However, obtaining a real waveform of the mouse blood flow rate would necessitate a rescaling of the model. Some estimation of mean flow characteristics can be obtained from our result plots since they represent non dimensional values versus the Hartmann number. Even if the magnetic fields used for small animals imaging are a lot greater than those used for humans, their effect would remain negligible since the mice vessels, their cardiac output and their flow rate are a lot smaller than those of humans. 
For example, the flow in a human aorta ( $1 \mathrm{~cm}$ radius) subjected to a $1.5 \mathrm{~T}$ magnet would yield a Hartmann number of 0.17 , whereas (considering that mice and human blood have the same viscosity and conductivity), the flow in the mouse aorta $(0.6 \mathrm{~mm}$ radius according to [21]) in a $10 \mathrm{~T}$ magnet would produce $H a=0.07$, thus inducing a mean flow rate reduction smaller than $0.1 \%$.

\subsection{On sum convergence}

In order to numerically evaluate the velocity and flow rate expressions which contain infinite sums, it was necessary to define a limit in order to sum over a finite number of terms. In other words, it was essential that we examine the sum convergence to define a stop point after which the added terms would not significantly modify the obtained sum.

Sum over $\boldsymbol{k}$. Referring to equation (6) while knowing that,

$$
\frac{u_{k}}{g_{k}}=2 \sum_{n=1}^{\infty} \frac{J_{0}\left(\lambda_{n} \tilde{r}\right)}{\lambda_{n} J_{1}\left(\lambda_{n}\right)} \quad \frac{1}{i \omega_{k} \frac{a^{2}}{\nu}+\lambda_{n}^{2}+H_{a}^{2}}<1,
$$

is uniformly limited and decreasing with $k$, it could be assumed that if after a certain value $K$ the harmonics $g_{k}$ for $|k|>K$ become negligible relatively to the other harmonics, the $u_{k}$ for $|k|>K$ will also be negligible relatively to all other $u_{k}$ for $|k|<K$. Therefore extending the sum to include all significant $g_{k}$ ensures that all significant $u_{k}$ are included. A harmonic $g_{k}$ is said to be significant if it is necessary to the accurate reconstruction of $g(t)$. It suffices then to find $K$ such as,

$$
g_{(K)}(t)=\sum_{k=-K}^{K} g_{k} e^{i \omega_{k} t} \approx g(t)
$$

Figure $9\left(\right.$ a) compares the pressure gradient curve to various plots of $\frac{\eta}{a^{2}} g_{(K)}$ for different values of $K$. It can be clearly noted that beyond $K=7$ the pressure gradient is fitted quite well.

In order to evaluate the gradient's fitting precision for a given $K$, we calculate a normalized mean error defined as,

$$
N M E(K)=\frac{1}{M} \sum_{i=1}^{M}\left|\frac{\left(-\frac{\partial p}{\partial z}\right)_{i}-\left(\frac{\eta}{a^{2}} g_{(K)}\right)_{i}}{\left(-\frac{\partial p}{\partial z}\right)_{i}}\right|,
$$

where $-\frac{\partial p}{\partial z}$ is calculated by (12), $M$ is the number of points of the regarded time functions and the index $i$ represents the $i^{\text {th }}$ point of the function (at time 


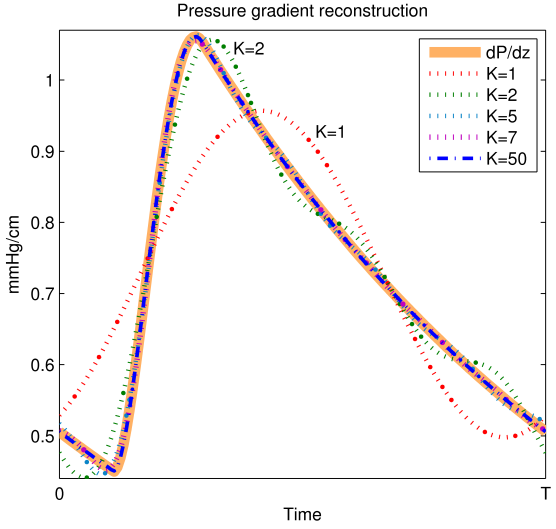

(a) Reconstructed pressure gradient curves for various values of $K$

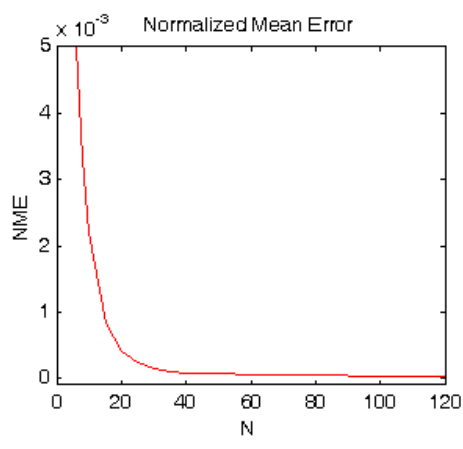

(b) Normalized mean error on the pressure gradient reconstruction

Figure 9. Pressure gradient reconstruction from $\frac{\eta}{a^{2}} g_{(K)}$

$\left.t_{i}\right)$

The evolution, in respect to $K$, of the normalized mean error is shown in figure 9(b), where a rapid decline of the NME can be observed, until it stabilizes after $K=50$.

To obtain the results presented in 4 we deemed acceptable to sum over $-50 \leq$ $k \leq 50$ only, with a $N M E(50)=6.81 \times 10^{-5}$. Note that to reduce the error to half its value we must extend the sum to $K=2000$, thus summing with 40 times the number of terms.

Sum over $n$. Having set the k sum limits, we then focus on the $\mathrm{n}$ sum. The number of terms $N$ necessary for the convergence of the sum over $n$ depends on the value of $H_{a}$ and increases with it. This can be observed in figure 10 which shows flow rate curves plotted for various values of $N$ with three $H_{a}$ numbers. It can be noted that for small $H_{a}$ the convergence is fast and is attained for $N=5$, whereas for large $H_{a}$ it's not until $N=50$ that we no longer observe any changes in the curve shapes. By calculating the NME between two curves for different values of $N$,

$$
\operatorname{NME}\left(N_{1}, N_{2}\right)=\frac{1}{M} \sum_{i=1}^{M}\left|\frac{\left(q_{(N 1)}\right)_{i}-\left(q_{(N 2)}\right)_{i}}{\left(q_{(N 2)}\right)_{i}}\right|,
$$

we find that for $H_{a}=20$, for example, going from $N=50$ to $N=1000$ only improves the result by $4 \times 10^{-5}$ i.e. $\operatorname{EMN}(50,1000)=4 \times 10^{-5}$, hence we deemed sufficient to set $N=50$.

The influence of $N$ can also be noted by comparison to Womersley and Sud's 


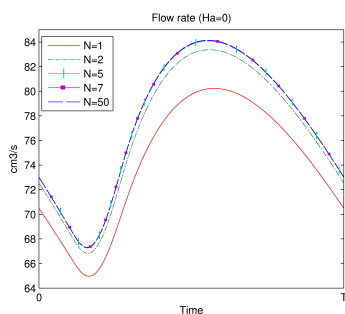

(a) $H_{a}=0$

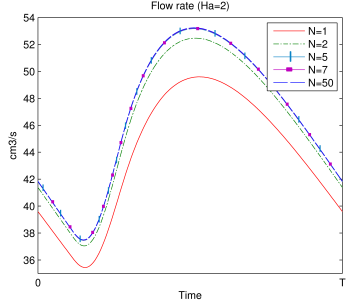

(b) $H_{a}=2$

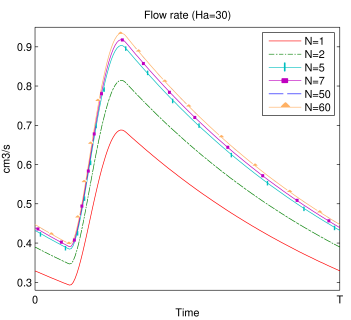

(c) $H_{a}=30$

Figure 10. Flow rate curves convergence for different values of $H_{a}$

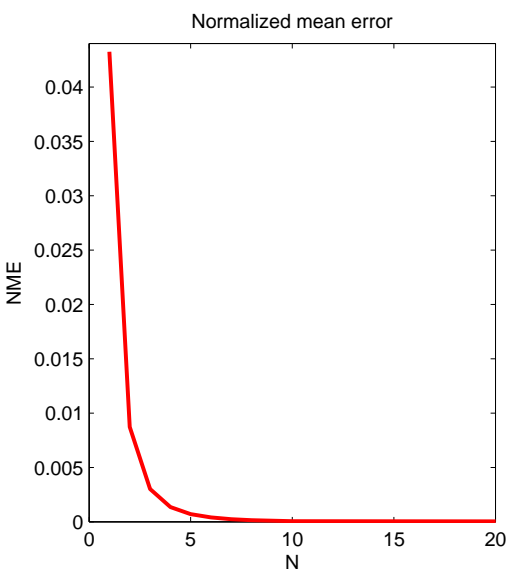

(a) Normalized mean error between flow rate curves calculated by (8) and those of Womersley given by (24) for $H_{a}=0$

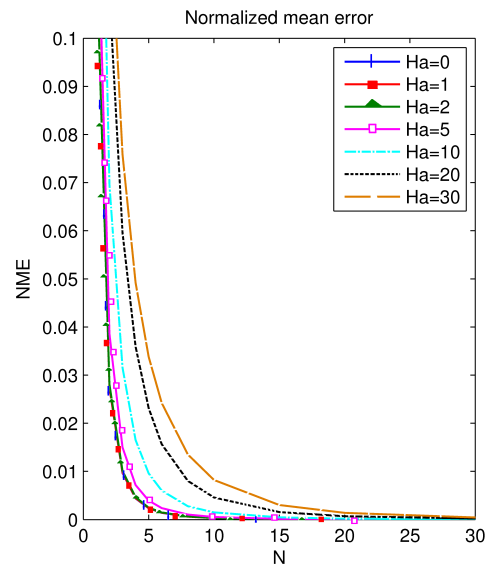

(b) Normalized mean error between flow rate curves calculated by (8) and those of Sud computed by (30) for various $H_{a}$

Figure 11. Normalized mean errors to other sinusoidal flows

sinusoidal flows. In fact the mean error rate between the curve $\left(H_{a}=0\right)$ and that of Womersley depends on the number of terms in the sum over $n$, and it decreases as that number increases (fig.11(a)). For example, for $N=5$ we get $N M E \approx 7 \times 10^{-4}$ and $N M E \approx 4.5 \times 10^{-5}$ for $N=50$. The same also applies for Sud's flow. Figure 11(b) shows the mean flow rates errors for various $H_{a}$. When $H_{a}$ increases, a larger $N$ is needed to ensure convergence. With $N=50$ we get an error of $9 \times 10^{-5}$ for $H_{a}=30$ (the same error is obtained for $N<15$ when $H_{a}=1$ ) while for $H_{a}<10$ the error remains $<2.7 \times 10^{-5}$.

\subsection{On induced magnetic fields}

The flow reduction depicted in our results does not accurately reflect the real movement retardation upon exposure to magnetic fields, especially very high 
ones. In fact, neglecting the induced fields produces an overestimation of the retardation and flattening effects. However it is noteworthy to mention that in real life situations like magnetic resonance imaging the involved Hartmann values are very small $(<<2)$ making this approximation quite acceptable, unless of course we are interested in computing induced voltages. For example, in the extreme case of a 15 Tesla magnet (i.e. $H_{a}=0.5$ for $a=0.3 \mathrm{~cm}$ ) the overestimation of the mean velocity reduction amounts to $3.5 \%$ only. Nevertheless, when dealing with larger vessels such as the aorta, one must be carful using this aproximation. For instance, neglecting the inductions in a $10 T$ field leads to a $10 \%$ overestimation, wherease considering a $15 T$ magnet intensity produces an additional $27 \%$ flow reduction compared to the exact solution.

\subsection{On magnetohydrodynamic blood flow in elastic vessels}

Studying magnetohydrodynamic blood flow in elastic vessels would be very interesting, especially since it would allow a close modeling of the ECG contaminating voltages, which are mainly generated in the aorta. Nevertheless, introducing wall motion in the model would impede attaining an analytical solution while using a physiological pressure gradient. Sud et al. [22] had proposed a flow rate expression for a simple sinusoidal pressure gradient in an elastic tube while neglecting the induced fields. A novel approach is currently in development [23], where the magnetic forces are incorporated in one-dimensional model equations. A numerical resolution of the established equations would produce flow results using a realistic pulsed pressure gradient while taking into account the induced electromagnetic fields.

\section{Conclusion}

In this work we studied the magnetohydrodynamic flow of blood in a rigid vessel in the presence of a static magnetic field. Even though some simplifying hypothesis were made, the originality of this work consists in applying a real physiological pressure gradient model. The gradient expression was obtained from a lumped model where a windkessel compliant module ensured the transition between ventricular flow and the rigid vessel input. The Fourier series based solution made it possible to evaluate our results using previous well established solutions for both steady or sinusoidal blood flows. As expected, a comparison with flows in the absence of any magnetic field showed the effects of a magnetic field in terms of flow reduction and velocity profile flattening. Moreover, a perfect match was attained when comparing to solutions derived in the presence of a magnetic field when inductions were neglected, while a comparison with the exact solution proved that the induced fields lessen the 
flow reduction. These calculations can be further improved by enhancing the lumped model and extending the magnetohydrodynamic equations to include induced fields.

\section{References}

[1] Keltner, J.R., Roos, M.S., Brakeman, P.R. and Budinger, T.F., 1990, Magnetohydrodynamics of blood flow. Magnetic Resonance in Medicine, 16, 139-149.

[2] Tasu, J.P., Mousseaux, E., Delouche, A., Oddou, C., Jolivet, O. and Bittoun,J., 2000, Estimation of pressure gradients in pulsatile flow from magnetic resonance acceleration measurement. Magnetic Resonance in Medicine, 44, 66-72.

[3] Gaffey, C.T. and Tenforde, T.S., 1981, Alterations in the rat electrocardiogram induced by stationary magnetic fields. Bioelectromagnetics, 2, 357-370.

[4] Tenforde, T.S., Gaffey, C.T., Moyer, B.R. and Budinger, T.F., 1983, Cardiovascular Alterations in Macaca Monkeys Exposed to Stationary Magnetic Fields: Experimental Observations and theoretical Analysis. Bioelectromagnetics, 4, 1-9.

[5] Chakeres, D.W., Alayar, K., Bouboulas, H. and Young, D.C., 2003, Effect of static magnetic field exposure of up to 8 Tesla on sequential human vital sign measurments. Journal of magnetic resonance imaging, 18, 346-352.

[6] Korchevskii, E. and Marochnik, L., 1965, Magnetohydrodynamic version of movement of blood. Biofizika, 10 (2), 371-373.

[7] Gold, R.R., 1962, Magnetohydrodynamic pipe flow Part 1. Journal of Fluid Mechanics, 13, $505-512$.

[8] Vardanyan, V.A., 1973, Effect of a Magnetic Field on Blood Flow. Biofizika, 18 (3), 491-496.

[9] Sud, V.K., Suri, P.K. and Mishra, R.K., 1974, Effect of magnetic field on oscillating blood flow in arteries. Studia biophysica, 46 (3), 163-172.

[10] Kinouchi, Y., Yamagushi, H. and Tenforde, T.S., 1996, Theoretical analysis of magnetic field interaction with aortic blood flow. Bioelectromagnetics, 17 (1), 21-32.

[11] El-Shehawy, E.F., Elbarbary, E.M.E., Afifi, N.A.S. and El Shahed, M., 2000, MHD flow of an elastico-viscous fluid under periodic body acceleration. International Journal of Mathematics $\mathcal{G}_{\mathcal{G}}$ Mathematical Sciences, 23 (11), 795-799.

[12] Spiegel, M. R., 1968, Schaum's Mathematical Handbook of Formulas and Tables. Mcgraw-Hill.

[13] Olufsen, M. and Nadim, A., 2004, On deriving lumped models for blood flow and pressure in the systemic arteries. Mathematical Biosciences and Engineering, 1 (1), 61-80.

[14] Westerhof, N., Elzinga, G. and Sipkema,P., 1971, An artificial arterial system for pumping hearts. Journal of Applied Physiology, 31, 776-781.

[15] Ottesen, J., Olufsen, M. and Larsen, J., 2004, Applied Mathematical Models in Human Physiology. Society for industrial and applied mathematics (Philadelphia).

[16] Segers, P. and Verdonck, P., 2000, Role of tapering in aortic wave reflection: hydraulic and mathematical model study. Journal of Biomechanics, 33, 299-306.

[17] Comolet, R., 1984, Biomécanique circulatoire. Masson (Paris).

[18] Abramowitz, M. and Stegun, I., 1964, Handbook of Mathematical Functions. Dover (New York).

[19] Womersley, J.R., 1955, Method for the calculation of velocity, rate of flow, and viscous drag in arteries when the pressure gradient is known. Journal of Physiology, 127, 553-563.

[20] Stergiopulos, N., Westerhof, B. and Westerhof, N., 1999, Total arterial inertance as the fourth element of the windkessel model. American Journal of Physiology - Heart Circulation Physiology, 276, 81-88.

[21] Segers, P. , Georgakopoulos, D. , Afanasyeva, M. , Champion, HC. , Judge, DP. , Millar, HD. Verdonck, P. , Kass, DA., Stergiopulos, N., Westerhof, N., 2005, Conductance catheter-based assessment of arterial input impedance, arterial function, and ventricular-vascular interaction in mice. American Journal of Physiology - Heart and Circulatory Physiology, 288(3) , 1157-1164.

[22] Sud, V.K , Suri, P.K and Mishra, R.K. 1978, Laminar flow of blood in an elastic tube in the presence of magnetic field. Studia biophysica, 69 (3), 175-186.

[23] Abi Abdallah, D. 2007, A new method for improving cardiac MRI synchronization, modeling the MagnetoHydrodynamic effect. PhD thesis, University of Technology of Compiègne. 